\title{
Wikileaks: el poder entre bastidores ${ }^{1}$
}

\author{
Marcello SERRA \\ Universidad Complutense de Madrid \\ serra.marcello@gmail.com
}

Recibido: 23/07/2011

Aceptado: 18/10/2011

Resumen El artículo razona sobre el impacto de Wikileaks y otros proyectos similares en el mundo político y en la esfera de la información. Siguiendo la idea de que el verdadero contenido de Wikileaks está en su forma, estas cuestiones son abarcadas prescindiendo del valor de las filtraciones. En particular, se analiza cómo la exposición del espacio entre bastidores de ciertas esferas del poder pueda delinear la posibilidad de una vigilancia de las instituciones por parte de los ciudadanos. En todo esto, resulta central la capacidad de solucionar el posible overload informativo producido por una gran cantidad de filtraciones, un fenómeno que, por un lado, comporta unas modificaciones en el periodismo de investigación $\mathrm{y}$, por otro, apela a la capacidad interpretativa del moderno general intellect.

Palabras clave: Wikileaks, privacidad, panopticum, general intellect, periodismo de investigación

\section{Wikileaks: the Backstage of Power}

\begin{abstract}
The article discusses the impact of Wikileaks and other similar projects on the world of politics and information. These themes are tackled putting aside the value of the leaks, following the idea that the real content of Wikileaks is its form. In particular, it analyses how exposure of the backstage in certain spheres of power could give citizens the ability to invigilate the institutions. One important theme is the ability to solve the possible information overload produced by the large quantity of leaks; a phenomenon which, on one hand, causes changes in investigative journalism and, on the other, appeals to the interpretative ability of the modern general intellect.
\end{abstract}

Keywords: Wikileaks, Privacy, Panopticon, General Intellect, Investigative Journalism

\section{Referencia normalizada}

SERRA, Marcello (2012): "Wikileaks: el poder entre bastidores". Estudios sobre el mensaje periodístico. Vol. 18, núm. 1, págs.: 211-220. Madrid, Servicio de Publicaciones de la Universidad Complutense.

Sumario: 1. Reflexión sobre Wikileaks. 2. Conceptos y efectos de la transparencia. 3. El cambio de ritmo comunicativo. 4. Inteligencia conectiva y colectiva. 5. Referencias bibliográficas.

\section{Reflexión sobre Wikileaks}

Digámoslo desde el principio: si merece la pena detenerse sobre el fenómeno Wikileaks desde el punto de vista de las ciencias de la comunicación no es seguramente por los documentos que difunde. Y digo esto no tanto por la escasa relevancia que, a veces, parecen tener sus filtraciones. Al contrario, la razón es que su verdadero contenido reside en su forma. Una vez más, en otras palabras, el medio es el mensaje, y es en la estructura de la mediación que hay que buscar la causa de sus efectos culturales. Aunque pueda parecer extraño, quiero empezar mi razonamiento con un poco de arqueología del problema.

${ }^{1}$ Este trabajo es resultado de estudios realizados en el marco del proyecto de I+D El fenómeno Wikileaks en España: un análisis semiótico y mediológico, dirigido por el catedrático Jorge Lozano Hernández (referencia CSO2011-23315) 
En The Americans: The Democratic Experience, de Daniel Boorstin, hay un pequeño capítulo, titulado "El declive de la unicidad y del secreto", en el que se cuenta una historia de hace cuarenta años. En enero de 1972, James Reston del New York Times se preguntaba por qué tantos documentos secretos del gobierno se estaban filtrando y llegaban a manos de la prensa. En aquel entonces, se trataba de una pregunta de actualidad en Estados Unidos ya que acababan de publicarse, de forma no autorizada, los famosos Pentagon Papers, en los que se revelaban unos secretos militares relativos a la guerra de Vietnam. Reston contestaba a esta pregunta afirmando que la verdadera fuente de las filtraciones era Chester Carlson, el inventor de la fotocopiadora. En otras palabras, reconducía el problema a una cuestión de impacto de una tecnología sobre la cultura, razonando según criterios propios del así llamado determinismo tecnológico. Puesto que, con la máquina fotocopiadora, todo el mundo podía hacer una copia instantánea de cualquier documento y de la verdad, ya no era posible estar seguros de que un documento fuera único. Al respecto, BoORSTIN señalaba también una consecuencia interesante al observar que los funcionarios del gobierno habían devenido recelosos para expresar con honestidad sus opiniones por escrito.

Este último es un asunto sobre el que prefiero volver más adelante. De momento quedémonos en Estados Unidos y en el mismo año, 1972, cuando Carl Bernstein y Bob Woodward se hacen protagonistas de uno de los casos más importantes y famosos de la historia del periodismo de investigación. A través de sus artículos, publicados en las páginas del Washington Post, se viene a saber que el equipo de Richard Nixon había sido protagonista de una serie de delitos, entre los cuales se hallaba el robo de información en la sede del Comité Demócrata Nacional en el complejo de oficinas Watergate.

La prueba fundamental por la que Nixon será luego condenado era representada por unas grabaciones efectuadas por él mismo que demostraban cómo el Presidente de los Estados Unidos había obstruido a la justicia e intentado tapar el delito. Pero, ¿por qué Nixon dijo lo que dijo sabiendo que las conversaciones estaban siendo grabadas? La primera razón es que se sentía intocable, y siendo el presidente de los Estados Unidos es algo que se puede entender. La segunda es que no había entendido que la presencia de la tecnología podía transformar de privadas a públicas sus conversaciones y la situación en la que se encontraba.

Ahora bien, ¿qué tiene que ver todo este preámbulo con Wikileaks?

En primer lugar, aunque se trate de un fenómeno con sus especificidades y, en parte, cualitativamente diferente con respecto a lo que lo precede, es posible enmarcar algunos aspectos de Wikileaks en un proceso de larga duración que tiene que ver con la redefinición del espacio público y privado operada por los medios.

En segunda instancia, como ya he dicho, no me interesa razonar sobre el contenido de los documentos filtrados, sino sobre las cualidades 'formales' de Wikileaks y sus relaciones con otras tecnologías de la información. Al respecto, estoy bastante de acuerdo con la afirmación del director del New York Times, Bill Keller, cuando afirma que "Wikileaks no inventó una nueva era en periodismo, es más un síntoma de lo que está ocurriendo en los últimos años en Internet". En otras palabras, estoy convencido 
que razonar sobre Wikileaks significa razonar sobre el cambio de paradigma comportado por la redes, sobre las continuidades y sobre las discontinuidades con respecto al modelo precedente.

Desde un punto de vista tecnológico, Wikileaks es una plataforma que se basa en el intercambio anónimo de información y en su acumulación indefinida. Desde una perspectiva política, su consecuencia directa es la reiteración de un afortunado imperativo internetiano: access for all. Acceso para todos y, podríamos añadir en perfecta ética hacker (HIMMANEN 2002), a todo. De hecho, como afirma el periodista del Guardian Nick Davies, que ha sido uno de los interlocutores más importantes de Julian Assange en el último año, el principal problema que, al principio, encontraban los periódicos a la hora de colaborar con el fundador de Wikileaks era el hecho de que "se trataba básicamente de un hacker informático. Proviene de una ideología simplista, o al menos en esa etapa la tenía, que cree que toda información tiene que ser pública, que cualquier información es buena" (LEIGH y HARDING 2011: 131).

Es verdad que, a partir de la segunda mitad del 2010, la postura de Wikileaks ha cambiado mucho, ha aceptado el hecho de que las noticias fueran de alguna forma redactadas y hasta se ha convertido en algo parecido a una plataforma de publicación mainstream; sin embargo, más allá de las posturas e ideologías personales e incluso más allá de las efectivas prácticas de difusión de la información, la misma forma de Wikileaks vehicula un mensaje preciso, y el mensaje es que, mientras sea posible saber algo, lo que sea, entonces es justo que se haga público ${ }^{2}$. De hecho, una de las críticas recibidas por Wikileaks, sobre todo después de los cambios en su política, es no haber revelado todo lo que tiene y, lo que en parte es lo mismo, haber decidido filtrar ciertas informaciones (por ejemplo los famosos cables diplomáticos estadounidenses) a través de unos periódicos tradicionales, aceptando sus normas de tratamiento de la información y de newsmaking.

En este sentido, por un lado Wikileaks continúa con la tendencia propia del principio de reproductibilidad técnica ${ }^{3}$ de desvelar los secretos; por otro sigue con el proceso de erosión de la esfera privada iniciado con los medios de comunicación de masas y las tecnologías de vigilancia. Como escribe Daniel Domscheit-Berg, que fue el más estricto colaborador de Assange durante dos años y medio y es uno de los fundadores de la plataforma OpenLeaks, “¿qué es público y qué es privado? Nuestra intención era crear

${ }^{2}$ Que así estén las cosas me parece indirectamente confirmado por la percepción que de Wikileaks tienen también (por lo menos) algunas de las personas directamente involucradas en ello, como Daniel DomscheIT-BERG: "WL era una plataforma, una herramienta, un ejemplo de tecnología devota a una absoluta neutralidad, sin intervenciones" (DOMSCHEIT-BERG 2011: 150 , trad. mía).

${ }^{3}$ De hecho, el desvanecimiento del aura de la obra de arte descrito por Walter BENJAMIN (1973) depende de procesos análogos. La lejanía necesaria a la presencia del aura corresponde a una distancia cultural, psicológica y comunicativa de parte de unas personas privadas tanto de la posibilidad de acceder a la visión de las obras como de los códigos necesarios para entenderlas. En el caso del arte la reproductibilidad hace desvanecer al misterio, en el caso de la información los que desaparecen son los secretos. O por lo menos, esto es lo que parece. 
controversia alrededor de esta cuestión" (DOMSHEIT-BERG 2011: 50, trad. mía) ${ }^{4}$. De hecho, la ideología vehiculada por la forma de Wikileaks y sus epígonos es la de una transparencia en la que pierde valor la distinción entre espacio público y privado o, mejor dicho, entre espacio público y espacio entre bastidores, ese espacio donde, por ejemplo, los actores políticos y diplomáticos preparan sus performances públicas (u oficiales).

Como nos ha enseñado Joshua MEYRowitz (1985), esta pérdida de distinción es posible porque la relación tradicional entre ambiente físico y situación social está comprometida por la difusión de los medios de comunicación electrónicos: los espacios informativos transcienden los espacios físicos y una habitación cerrada no es garantía de privacidad, de intimidad o de secreto. Pero, más en general, en la cultura de Internet es el concepto mismo de privacidad que aparece como obsoleto.

Al respecto es interesante la reflexión que 0100101110101101.org, ahora conocidos como Eva y Franco Mattes, desarrollaba ya en el 2001 con el proyecto life_sharing. Transposición de 'file sharing', en life_sharing 01.org se dejaba que, mediante conexión remota, todos los que los quisieran accediesen libremente y en tiempo real a los datos contenidos en su ordenador principal: al software, al sistema operativo, al disco duro y, en consecuencia, a correos y fotografías personales, hecho especialmente significativo si consideramos que, en aquel entonces, sus identidades eran desconocidas. Ellos mismos comentaban así este proyecto:

La guerra del secreto (criptografía, anonimato, etc.) es desafortunadamente una batalla perdida. Las grandes corporations tendrán siempre a su disposición instrumentos más sofisticados del usuario común, más capacidad de cálculo, más control a través de los satélites. Es posible mantener el anonimato sólo a un nivel superficial. Después de un cierto nivel ya no es posible. Toda transacción económica, cualquier compra o venta, cualquier relación humana está basada en la documentación. Cuanto más vaya dependiendo la sociedad del ordenador, más facilitado será este proceso. La fuerza real de 0100101110101101.ORG es su visibilidad. La única manera de evitar el control es el exceso de datos, el amontonamiento y la multiplicación de datos hasta el punto que devenga extremamente difícil aislarlos e interpretarlos (FULLER 2001: web, trad. mía).

\section{Conceptos y efectos de la transparencia}

Lo que pasa ahora con Wikileaks -y aún más con la proliferación de todas las otras plataformas inspiradas en Wikileaks que hemos visto nacer en los últimos meses- es que, perdida la guerra del secreto, la sociedad civil parece tener ciertas posibilidades para ganar algunas batallas por la transparencia. Las instituciones son víctimas del mismo proceso de erosión del espacio privado efectuado por parte de los medios de comunicación que afecta al ciudadano, y también vemos perfilarse un nuevo tipo de vigilancia: a la vigilancia de los ciudadanos por parte del Estado y a la de los consumidores por parte de las empresas se añade la vigilancia de las instituciones por parte de los ciudadanos.

${ }^{4}$ Y más adelante sigue así: “Además, estábamos convencidos de que ampliar poco a poco la frontera de lo aceptable era una forma de fortalecer el proyecto y constatamos que cada vez nos salíamos con la nuestra. Devenimos siempre más atrevidos. Nadie podía pararnos" (DOMSCHEIT-BERG 2011: 50, trad. mía). 
Entre otras cosas, esto tiene un efecto directo sobre la autoridad de los gobiernos. En primer lugar, hay un efecto que prescinde completamente de la relevancia de las informaciones filtradas: simplemente, el hecho de revelar, aunque sólo en parte, el espacio entre bastidores donde los gobiernos se preparan para luego ejercer el poder, no puede dejar de causar un golpe a su autoridad, es decir a la legitimidad, la justificación y el derecho de ejercer ese poder. La situación es análoga al padre o la madre que pierden autoridad frente a su hijo cuando su pareja le contesta, delante de este último, sus decisiones educativas: si se muestran las incertidumbres (o peor, la deshonestidad) del espacio entre bastidores, los actos oficiales parecerán cuestionables.

En segundo lugar, el 'quién sabe qué sobre quién' es cada vez más una cuestión de bases de datos informáticas. Quién gestiona esas bases de datos y quién tiene o no acceso a ellas es algo estrechamente relacionado con la distribución del poder y, evidentemente, el hecho de afirmarse de una práctica de difusión de información reservada cambia esta distribución. Además, en esta primera infoguerra mundial ${ }^{5}$, quizás los ciudadanos no tengan a disposición instrumentos menos sofisticados de los que tienen las instituciones. En concreto, el arma más poderosa en su posesión es la conexión, la inteligencia conectiva que nace de la arquitectura de la red.

Uno de los problemas más patentes en toda la cuestión de Wikileaks es cómo sacar información de una cantidad de documentos enorme, desorganizada y, en muchos casos, irrelevante. Como observaba 01.org, en una sociedad altamente informatizada y en red "la única manera de evitar el control es el exceso de datos, el amontonamiento y la multiplicación de datos hasta el punto que devenga extremamente difícil aislarlos e interpretarlos". En otras palabras, siguiendo el principio por el que un exceso de información equivale a su ausencia, 01.org teoriza la difusión de información personal (verdadera, jno ficticia!) como táctica de invisibilidad. Pero, en principio, esto debería valer también para las instituciones, una multinacional o un gobierno. ¿O tal vez las cosas sean de otra forma?

Antes de contestar a esta pregunta, se me permita una digresión. Hace unos años un amigo, Tonino es su nombre, se fue a vivir a Londres donde, para ganarse la vida, trabajaba como repartidor, distribuyendo paquetes en moto por la ciudad. Un día le llegó a casa una multa por haber puesto el pie en el suelo en un cruce de tráfico; puesto que iba en moto, esto significaba que hubo un momento en el que se había parado en el cruce, ocupándolo cuando no se podía. ¿Pie en el suelo? A mi amigo Tonino la cosa le sonó ridícula y se dirigió hacia el puesto más cercano de policía municipal para que le quitaran la multa. ¿Como podían comprobar que él había efectivamente apoyado el pie en el suelo durante unos segundos? Según lo que contó después a los amigos, el oficial de la policía fue muy amable: le contestó que tenía todo el derecho de reclamar y que, si efectivamente no había cometido ninguna infracción, le quitarían la multa; sin embargo, tenía que advertirle que, en el caso de que reclamase oficialmente,

5 "La primera infoguerra ha comenzado. El campo de batalla es Wikileaks. Vosotros sois las tropas", escribió John Perry Barlow en un tweet que, reenviado por el mismo twitter de Wikileaks, ha dado la vuelta al mundo. 
ellos estarían obligados a reconstruir, a través de las imágenes grabadas por las miles de videocámaras presentes en Londres, todos sus movimientos de aquel día, a partir del momento en el que había salido de casa. Después de estas aclaraciones Tonino se hizo dos cuentas, sacó la cartera y pagó en efectivo.

Yo no sé cuánto de esta historia será verdad y cuánto mitografía, pero la anécdota toca algunos puntos importantes para nuestro discurso. Tonino paga y, sobre todo, cambia su estilo de conducir porque, a diferencia de Nixon, tiene miedo. Él no sabe si la policía tiene realmente esas imágenes pero renuncia a recurrir y, desde aquel momento, presta mucha atención a las reglas del tráfico inglés. En otras palabras, asume inconscientemente el principio del panopticum de Jeremy Bentham, donde el vigilante se transforma en una entidad puramente semiótica, que cumple su función aunque nadie, efectivamente, esté controlando los prisioneros: Tonino no sabe si lo están vigilando, no ve a nadie haciéndolo, pero sabe que podrían y, entonces, obedece las reglas.

Como ya observábamos al principio citando a Boorstin y sus consideraciones sobre el papel de la fotocopiadora en las filtraciones de los Pentagon Papers, esto nos indica también que, después de la llegada de Wikileaks en el panorama de la información, todo apunta para que haya unos cambios de conducta en ciertos ámbitos por el temor de que ciertas informaciones se hagan públicas. Por ejemplo, el tono de algunos de los cables filtrados revela la despreocupación típica de la esfera privada. Al contrario, el temor de poder estar controlados puede que genere algo parecido a lo que pasa en situaciones como las instituciones totales estudiadas por Erwin Goffman, donde el aumento de la presión para interpretar un papel de forma correcta produce cierta tensión y rigidez de comportamiento. En otras palabras, la posibilidad de que el espacio entre bastidores del poder quede expuesto, a través de unas filtraciones, a la mirada pública, puede funcionar como dispositivo panóptico invertido, asumido por las esferas del poder.

\section{El cambio de ritmo comunicativo}

En un sentido parecido se puede pensar también en la idea de MCLUHAN según la cual el poder innovador de las nuevas tecnologías ${ }^{6}$ opera a nivel de las estructuras y que es en este nivel donde su mensaje se expresa (y en cierto sentido se agota): "porque, el 'mensaje' de cualquier medio o tecnología es el cambio de escala, ritmo o patrones que introduce en los asuntos humanos" (McLuHAN 1996: 30). Según este principio, el cambio de ritmo comunicativo impuesto por un nuevo medio de comunicación pone en crisis el funcionamiento de las estructuras precedentes: "poned un teléfono dentro de una universidad", afirma, "y la haréis añicos. [...] Poned un teléfono dentro de cualquier burocracia, y la derrumbaréis" (cit. en GAMALERI 1976: 30, trad. mía). De la misma forma, la presencia de Wikileaks comporta una reorganización del funcionamiento de una serie de otros campos, que van desde la diplomacia al sistema de la información.

${ }^{6}$ Con esta expresión me refiero, evidentemente, a la novedad de las tecnologías en el momento en el que se introducen en la sociedad y no a las que hoy se consideran corrientemente como new media (telefonía móvil, internet, etc.) 
En particular, éste último ve cuestionadas su ética, sus criterios de noticiabilidad, sus rutinas de trabajo y su capacidad de digerir la información proporcionada por fuentes como Wikileaks. Esto nos hace volver a la cuestión del overload informativo, que habíamos dejado pendiente. Para tener una idea del efecto que la masa de datos en manos de Wikileaks pueda tener en un periódico tradicional me parece interesante ir a una fuente directa, la crónica que los periodistas del Guardian David LEIGH y Luke HARDING hacen de la reacción que tuvo la redacción a la llegada de las filtraciones sobre Afganistán:

[...] las propias bases de datos parecían enormes, confusas e imposibles de gobernar; un bosque impenetrable de jerga militar. Davies, a esta altura agotado por la larga jornada, empezó a preguntarse si realmente incluían algo que tuviera valor periodístico. [...] Reunido alrededor de una mesa, el equipo del Guardian repasaba las dificultades técnicas. David Leigh se mostraba irascible: 'Es como encontrar pequeñas pepitas de oro en medio de una montaña de datos - protestaba-, ¿cómo vamos a saber nunca si esconde buenas noticias?'. La respuesta a esta pregunta puso las viejas manos del Guardian sobre una empinada curva de aprendizaje en la que iban dominando los métodos más modernos. Harold Frayman, el experto técnico, resolvió estos problemas: improvisó rápidamente una base de datos a escala real. Como la de Google o de avanzados buscadores de noticias como el LexisNexis, la base de datos de Frayman podía buscar por fecha, por palabra clave o por cualquier frase puesta entre comillas. [...] Leigh también empezó a animarse: ‘¡Ahora estos datos empiezan a decirme algo!' exclamó.

A Leigh le presentaron otro especialista del Guardian, Alastair Dent: 'Alastair es nuestro analizador de datos', le dijeron. 'Ni siquiera sabía que existía un trabajo así'. Pronto lo pusieron al día. El proyecto Wikileaks estaba produciendo nuevos tipos de datos, así que necesitaban ser extraídos con nuevos tipos de periodismo. Dant explicó que podía convertir las estadísticas de los miles de explosiones de bomba grabadas en los diarios de guerra de Afganistán en una visualización gráfica animada a medida[...]. Era una interpretación que aportaba cierto grado de inteligibilidad a una guerra que, por lo demás, resultaba rudimentaria y mal narrada.

El experto visual clave resultó ser Simon Rogers, el editor de datos del Guardian. 'Tú eres bueno con las hojas de cálculo, ¿no?', le preguntaron. 'Sí, pero esta hoja de cálculo se las trae', respondió. Después de trabajar con aquellas hojas, concluyó: 'A veces la gente habla de que internet acabará con el periodismo. La historia de Wikileaks es una combinación de las dos cosas: los conocimientos del periodismo tradicional y el poder de la tecnología, unidos para contar una historia alucinante. En el futuro, tal vez el periodismo de datos no parezca nuevo ni sorprendente; por ahora, lo es. El mundo ha cambiado y son los datos los que lo han cambiado' (LeIGH y HARDing 2011: 119-126).

Este testigo, en el que se observa perfectamente la idea mcluhaniana del cambio en la organización interna de una institución causado por una innovación tecnológica, obliga también a reflexionar sobre el periodismo de investigación. Brevemente, podemos decir que éste se compone de tres fases. En la primera se descubren unos hechos, en la segunda se controlan a través de un contraste de las fuentes y de los datos mismos, en la tercera se introducen en un discurso comprensible.

Geert LoovinK y David RiEMENs ya han notado cómo Wikileaks sigue sólo una parte del proceso. Seguramente se ocupa de la primera fase, la de descubrir los hechos, y pretende hacer la segunda, aunque los criterios adoptados no parece que sean muy 
rígidos ${ }^{7}$. Por otro lado, no se interesa mínimamente de la tercera que, sin embargo, es fundamental. La razón de su importancia es que es ahí que un simple acontecimiento pasa a ser un hecho, es decir que se transforma en algo más que un acontecimiento aislado y se convierte en parte de una intriga, de un relato coherente que lo explica, lo introduce en una cadena causal que le otorga sentido (lo que es, ni más ni menos, lo que pretendía poder hacer el policía inglés que amenazaba a mi amigo Tonino).

Más allá de la búsqueda de visibilidad, la ausencia de esta fase es una de las razones más importantes de la colaboración de Wikileaks con los periódicos, quienes podían realizarla. Sin embargo, considerando que la cantidad de información reservada que se irá publicando está destinada a aumentar de forma exponencial, esta tarea interpretativa no podrá ser de pertinencia exclusiva de los periodistas. Además, se trata de una cuestión que está muy relacionada con la difusión y la recepción de las informaciones por parte de la opinión pública. Evidentemente, los periódicos, y aún más los grandes periódicos, están interesados sólo en un determinado tipo de noticias (las que satisfacen a sus criterios de noticiabilidad) y no pueden prestar atención a la mayoría de las filtraciones ${ }^{8}$.

\section{Inteligencia conectiva y colectiva}

Una solución a estos problemas intentan proporcionarla proyectos como Microleaks o Kanarileaks, dedicados a filtraciones locales, o el más ambicioso Openleaks, creado

\footnotetext{
${ }^{7}$ Al respecto, esto es lo que cuenta Domscheit-Berg: "Otro tema era el de nuestros 'controles de autenticidad' -un engaño que me he forzado a mí mismo llevar adelante en cientos de entrevistas-. Hasta finales del 2009, nadie excepto Julian y yo cotejó la vasta mayoría de los documentos recibidos. Hablando estrictamente, no estábamos mintiendo cuando decimos que teníamos un equipo de alrededor de 800 voluntarios a nuestra disposición. Pero olvidamos mencionar que no teníamos ningún mecanismo para integrarlos en nuestra dinámica de trabajo. Ninguno de ellos podía acceder al material que recibimos. En cambio, Julian y yo normalmente controlábamos si los documentos habían sido manipulados tecnológicamente y hacíamos unas búsquedas en Google para ver si nos parecían genuinas. Podíamos sólo esperar que las cosas resultasen bien. Al parecer desarrollamos un muy buen olfato para lo que era auténtico y lo que no era; por lo menos hasta donde sé, no cometimos ningún error importante. Pero podríamos haberlo hecho (DOMSCHEIT-BERG 2011: 178-179, trad. mía).

8 De hecho, esto se convirtió en un problema para el mismo Wikileaks en el momento en el que empezó a configurarse como una plataforma de publicación: "El verdadero problema de Wikileaks fue que intentó hacer demasiadas cosas al mismo tiempo. WL gestionó solo todo el proceso de traición de secretos digitales. Las fuentes colgaban los documentos; el equipo de Wikileaks los limpiaba de metadatos, verificaba los envíos y redactaba unas notas adjuntas sobre el contexto. Al final, todo eso se publicaba en su página web.

Pero llegó un momento en el que ya no nos era posible llevar a cabo todas esas tareas. Simplemente, nos llegaban demasiados documentos y habríamos necesitado cientos de voluntarios profundamente involucrados para salir adelante. Así pues, estábamos obligados a tomar decisiones. ¿Qué filtraciones deberían ver la luz del día y cuáles permanecer sin publicar en servidores esparcidos por el mundo? Estábamos sobrecargados" (DOMSCHEIT-BERG 2011: 218. trad. mía).
} 
por unos antiguos miembros de Wikileaks y que propone una estrategia alternativa de difusión de las filtraciones. En lugar de dedicarse a la publicación, Openleaks intenta hacer llegar las filtraciones al receptor cada vez más adecuado, sea éste un periódico, una $\mathrm{ONG}$, un sindicato u otro tipo de organización (y respetando, en caso de que haya una, la indicación de la fuente con respecto al destinatario).

Retomando algunas observaciones de Franco 'Bifo' BERARDI (2010), podemos notar cómo todo esto es parte de un proceso más amplio. En general, como soporte de los proyectos que se dedican a filtrar información secreta o reservada se ha creado una creciente inteligencia conectiva formada por trabajadores cognitivos de distinto tipo: técnicos de hardware, programadores, periodistas que trabajan juntos en el mismo proyecto ${ }^{9}$. Esta inteligencia conectiva ${ }^{10}$ que trabaja en las primeras dos fases de la investigación debe encontrar una inteligencia colectiva a la altura de las tareas impuestas por la tercera. El overload informativo que ya vemos llegar va a ser un reto al que nuestra sociedad podrá responder sólo transformando la cantidad de las conexiones de red en calidad, profundidad, lucidez interpretativa. En otras palabras, utilizando la dispersa pero poderosa fuerza cognitiva de nuestro moderno general intellect.

\section{Referencias bibliográficas}

BENJAMIN, Walter (1973): La obra de arte en la época de su reproductibilidad técnica, en id., Discursos interrumpidos I. Madrid, Taurus.

9 Así escribe Bifo, con tonos más politizados que los de este artículo: "El acontecimiento orquestado por Assange es la irradiación de la fuerza creativa del intelecto general. La lección de Wikileaks no está tanto en los contenidos revelados -ya sabíamos que a los diplomáticos se les paga por mentir y que a los militares se les paga por disparar contra los civiles- cuanto en la activación de la solidaridad, complicidad y colaboración independiente entre cognitarios, entre trabajadores cognitivos de distinto tipo: técnicos de hardware, programadores, periodistas que trabajan juntos y comparten el mismo objetivo de desestabilizar el poder totalitario. A partir de esta lección, los rebeldes encontrarán su camino hacia la auto-organización del intelecto general" (BERARDI 2010: web). Siguiendo este razonamiento, en el movimiento de lucha para la transparencia del cual Wikileaks es la bandera, veríamos a la obra un general intellect (una inteligencia conectiva-colectiva) que se propone como carden de una esfera pública multitudinaria, de alguna forma antitética a la esfera pública basada en el Estado y en su monopolio de la decisión política. Sin intentar ahora establecer vínculos causales -una operación que desbordaría los límites de este artículo-, que esto sea parte del espíritu del tiempo se puede observar en el imponente éxito del movimiento del 15M aquí en España.

10 Curiosamente, los fenómenos de inteligencia conectiva que hemos visto nacer con el desarrollo de las redes a menudo han sufrido cierta falta de estesía, cierta ausencia de corporeidad e incluso de reconocibilidad. En este sentido, desde el punto de vista del imaginario es curioso observar cómo Assange acabe por representar, guste o no, el cuerpo de una inteligencia conectiva. En la pauta del cuerpo del poder de Hobbes, la figura de Assange, por muy dilacerada, parece constituir una especie de cuerpo de contrapoder multitudinario. 
BERARDI, Franco (2010): "Seré sintético", en Multitud. Colaboratorio de singularidades simbiontes, en: http://colaboratorio1.wordpress.com/2010/12/24/sere-sintetico-franco-berardi-bifo/ [fecha de consulta: 09/07/2011]

BOORSTIN, Daniel J. (1973): The Americans: The Democratic Experience. New York, Vintage.

DOMSCHEIT-BERG, Daniel (2011): Inside Wikileaks: My Time With Julian Assange at The World's Most Dangerous Website. New York, Crown.

FULLER, Matthew (2001): "Data Nudism. An Interview with 0100101110101101 .ORG about life_sharing", en Gallery 9 / Walker Art Center, en: http://www.walkerart.org/gallery9/lifesharing/ [fecha de consulta: 09/07/2011]

GAMALERI, Gianpiero (1976): La galassia McLuhan. Il mondo plasmato dai media? Roma, Armando.

HIMMANEN, Pekka (2002): La ética del hacker y el espíritu de la era de la información. Barcelona, Destino.

LEIGH, David y HARDING, Luke (2011): Wikileaks y Assange. Un relato trepidante sobre cómo se fraguó la mayor filtración de la historia. Barcelona, Deusto.

LOOVINK, Geert y RIEMENS, Patrice (2010): "Twelve thesis on Wikileaks", en €urozine: http://www.eurozine.com/articles/2010-12-07-lovinkriemens-en.html [fecha de consulta: 09/07/2011]

MCLUHAN, Marshall (1996): Comprender los medios. Las extensiones del ser humano. Barcelona, Paidós.

MEYROWITZ, Joshua (1985): No sense of place. The Impact of Electronic Media on Social Behevior. New York-Oxford, Oxford University Press. 